\title{
La combinación salinomicina/nicarbazina como anticoccidial en pollos de engorde
}

\author{
THE SALINOMYCIN/NICARBAZIN COMBINATION AS ANTICOCCIDIAL IN BROILERS
}

Casandra Zavala D. ${ }^{1}$, Eliana Icochea D., ${ }^{1,3}$, Nelly Cribillero C. ${ }^{1}$, Daniel Molina M. ${ }^{2}$

\section{Resumen}

Se evaluó la eficacia en el control de la coccidiosis de la combinación anticoccidial de salinomicina/nicarbazina, suministrado en el alimento a una dosis de $40 \mathrm{ppm}$, por medio de la evaluación de parámetros productivos en pollos de engorde desafiados experimentalmente con cepas locales de coccidias y criados hasta los 42 días de edad. Se usaron 450 pollos de engorde de la línea Cobb Vantress 500, divididos en tres grupos experimentales con 6 repeticiones de 25 aves. G1: aves no desafiadas y tratadas con la combinación anticoccidial, G2: aves desafiadas y tratadas con la combinación anticoccidial, y G3: aves desafiadas y no tratadas. Se determinó el peso vivo, la ganancia de peso, el consumo de alimento, el índice de conversión alimenticia, el índice de eficiencia productiva europeo y la viabilidad. Las aves de los grupos G2 y G3 fueron desafiadas a los 14 días con $1 \mathrm{ml}$ de inóculo de coccidias conteniendo ooquistes esporulados de Eimeria acervulina $\left(10^{5}\right)$, E. maxima $\left(3 \times 10^{2}\right)$ y E. tenella $\left(10^{4}\right)$. Al final del estudio se obtuvo un peso vivo promedio de: 2650.4, 2431.6 y 2111.6 g; ganancia acumulada de peso: $2604.1,2384.0$ y 2063.8 g; conversión alimenticia: $1.53,1.61$ y 1.65; e índice de eficiencia productiva: 408.67, 355.94 y 282.00 para G1, G2 y G3, respectivamente. Todos los valores mostraron diferencia significativa $(\mathrm{p}<0.05)$. Se obtuvo un menor, pero no significativo porcentaje de viabilidad en G3 (mediana 94\%), en comparación con G1 y G2 (medianas 100\%). Se concluye que la combinación anticoccidial (salinomicina/nicarbazina) fue eficaz en el control de la coccidiosis al obtenerse mejores parámetros productivos de los grupos tratados.

Palabras clave: salinomicina; nicarbazina; coccidiosis; parámetros productivos; pollos de engorde

\footnotetext{
${ }^{1}$ Laboratorio de Patología Aviar, Facultad de Medicina Veterinaria, Universidad Nacional Mayor de San Marcos, Lima, Perú

${ }^{2}$ Ilender Perú S.A., Lima, Perú

${ }^{3}$ E-mail: micochead@unmsm.edu.pe
}

Recibido: 5 de noviembre de 2017

Aceptado para publicación: 8 de mayo de 2018 
The efficacy in the control of coccidiosis of the anticoccidial combination of salinomycin / nicarbazin, supplied in the feed at a dose of $40 \mathrm{ppm}$, was evaluated through changes in productive parameters in broilers challenged experimentally with local strains of coccidia and raised up to 42 days of age. Broilers of the Cobb Vantress 500 line $(n=450)$ were distributed into three experimental groups with 6 repetitions of 25 birds each. G1: chicks not challenged and treated with the anticoccidial combination, G2: chicks challenged and treated with the anticoccidial combination, and G3: chicks challenged and untreated. Body weight, body weight gain, feed consumption, feed conversion index, European productive efficiency index and the viability were determined. Broilers of groups G2 and G3 were challenged at 14 days with $1 \mathrm{ml}$ of coccidia inoculum containing sporulated oocysts of Eimeria acervulina $\left(10^{5}\right)$, E. maxima $\left(3 \times 10^{2}\right)$ and E. tenella $\left(10^{4}\right)$. Results showed body weight: 2650.4, 2431.6 and $2111.6 \mathrm{~g}$; accumulated weight gain: 2604.1, 2384.0 and $2063.8 \mathrm{~g}$; feed conversion: $1.53,1.61$ and 1.65 ; and productive efficiency index: $408.67,355.94$ and 282.00 for G1, G2 and G3, respectively. All values showed significant differences $(p<0.05)$. A lower but not significative percentage of viability was observed in G3 (median 94\%) as compared to G1 and G2 (median 100\%). It is concluded that the anticoccidial combination (salinomycin / nicarbazine) was effective in the control of coccidiosis as better productive parameters were obtained in the treated groups.

Key words: salinomycin; nicarbazin; coccidiosis; productive parameters; broilers

\section{INTRODUCCIÓN}

La coccidiosis es una enfermedad de presentación universal en pollos de engorde, en especial bajo condiciones intensivas de crianza. La forma clínica ocasiona pérdidas económicas por mortalidad y la forma subclínica, que es la más importante, por los bajos rendimientos productivos, expresados en retraso del crecimiento, pobres índices de conversión alimenticia, baja uniformidad y mala pigmentación de patas (Conway y McKenzie, 2007). Las pérdidas financieras en la industria avícola como resultado de la coccidiosis a nivel mundial han sido estimadas en cerca de US\$ 3 billones, las cuales principalmente se basan en gastos por medicación, tanto profiláctica como terapéutica, así como por las mermas en los parámetros productivos(Williams, 1999; Dalloul y Lillehoj, 2006).
El uso de programas anticoccidiales ha sido el método más efectivo para la prevención y control de la coccidiosis en pollos de engorde, especialmente con la adición de drogas anticoccidiales en el alimento balanceado de las aves (Del Cacho y Bosch, 2014). Los programas pueden ser continuos, si se suministra un mismo producto anticoccidial en la campaña completa, o duales, si la droga anticoccidial es cambiada a mitad de campaña (Del Cacho y Bosch, 2014). La prevención de la coccidiosis tiene como objetivo minimizar el daño intestinal para evitar que se afecte la productividad. Los fármacos anticoccidiales se administran en la ración durante toda la campaña de crianza, con excepción del periodo de retiro previo al sacrificio (Sumano y Gutiérrez, 2010).

Hay una variedad de drogas disponibles en el mercado que han logrado un buen control de la coccidiosis, pero existen ciertos ries- 
gos como son los peligros de toxicidad, de residuos químicos en la carne que obligan a un periodo de retiro, y generación de cepas resistentes o de sensibilidad reducida a lo largo del tiempo (Keshavarz y McDougald, 1982). La industria avícola busca alternativas de prevención mediante productos más eficaces, con menor riesgo de toxicidad y de menor costo. En algunos países se ha utilizado con éxito la mezcla de dos o más productos anticoccidiales, que brindan una acción potenciada o sinérgica con menor toxicidad y mayor eficacia, por tener un mayor rango de actividad con un lento desarrollo de resistencia (Glazer et al., 1993). Adicionalmente, la combinación anticoccidial permite abarcar tanto las fases luminales como las intracelulares de las coccidias (Hernández y Petrone, 2005).

El objetivo del presente trabajo fue evaluar la eficacia en el control de la coccidiosis de la combinación de dos drogas anticoccidiales, salinomicina y nicarbazina, suministradas en el alimento a una dosis de $40 \mathrm{ppm}$, mediante la evaluación de los parámetros productivos de pollos de engorde hasta los 42 días de edad.

\section{Materiales y Métodos}

\section{Lugar de Estudio y Animales}

El estudio se realizó entre febrero y marzo de 2014 en el galpón experimental del Laboratorio de Patología Aviar de la Facultad de Medicina Veterinaria (FMV) de la Universidad Nacional Mayor de San Mar$\cos$ (UNMSM), ubicado en el distrito de San Borja, Lima, Perú. Se utilizaron 450 pollos machos de la línea Cobb 500 de un día de edad, procedentes de un mismo plantel de reproductoras libres de enfermedades. Para la crianza de los pollos se utilizaron equipos convencionales, bebederos tipo tongo y campana, comederos tipo bandeja y tolva, criadoras de calefacción a gas, cama de vi- ruta de madera, mangueras y válvulas de gas, nórdex, cercos para separación de corrales, termohigrómetros y balanzas digitales.

Las aves recibieron una dieta estándar para pollos de engorde a base de maíz y soya, dividida en dos fases nutricionales: inicio ( 0 21 días) y crecimiento (22-42 días). La dieta estuvo libre de antibióticos promotores de crecimiento. El alimento y agua de bebida fueron administrados ad libitum según los requerimientos nutricionales de la edad y tipo de manejo. Para el manejo de las aves experimentales se tomaron en cuenta las normas del Comité de Ética y Bienestar Animal (CEBA) de la FMV-UNMSM, que consideran los lineamientos estándares de la crianza comercial de aves (densidad, temperatura, humedad, etc.), para garantizar el normal crecimiento de los pollos y un mínimo de estrés.

\section{Anticoccidial}

El producto evaluado es una asociación de un anticoccidial ionóforo (salinomicina) y uno químico (nicarbazina) administrado en la ración de forma continua desde la recepción hasta los 35 días de edad (7 días de retiro) a razón de $0.4 \mathrm{~kg}$ por tonelada de alimento, lo cual implica 40 ppm de cada principio activo (dosis recomendada por el proveedor Ilender Perú SA).

\section{Tamaño Muestral}

El cálculo de tamaño muestral fue obtenido a partir de la fórmula de estimación para una diferencia de medias en grupos independientes, para lo cual se asumió una diferencia mínima a detectar de $50 \mathrm{~g}$ con respecto al peso corporal, con una desviación de $15 \mathrm{~g}$ en ambos grupos, un nivel de significancia de $0.05 \mathrm{y}$ un poder de prueba de $80 \%$. El resultado indicó un mínimo de cuatro unidades experimentales por grupo; no obstante, se trabajaron seis unidades experimentales por grupo para aumentar la probabilidad de obtener los resultados productivos deseados. 


\section{Diseño Experimental}

Los animales se distribuyeron en un diseño completamente aleatorio de tres tratamientos experimentales de 150 pollos, con 6 repeticiones de 25 pollos c/u. Se tuvo un área de $2.25 \mathrm{~m}^{2}$ por corral, haciendo una densidad aproximada de $33 \mathrm{~kg} / \mathrm{m}^{2}$ y un peso aproximado de saca de $2.8 \mathrm{~kg} /$ ave. Los grupos fueron: G1) tratado con la combinación anticoccidial salinomicina/nicarbazina y no infectado, G2) tratado con la combinación anticoccidial salinomicina/nicarbazina e infectado, y G32) no tratado e infectado.

\section{Desafío Experimental}

Se preparó un inóculo con cepas de campo de Eimeria spp con el fin de inducir mortalidad y signos clínicos de coccidiosis en las aves desafiadas. Luego de varios pasajes in vivo se obtuvo una dosis infectiva con un título estandarizado de ooquistes esporulados de E. acervulina $\left(10^{5} / \mathrm{ml}\right)$, E. maxima $\left(3 \times 10^{2} / \mathrm{ml}\right)$ y E. tenella $\left(10^{4} / \mathrm{ml}\right)$.

El desafío de las aves se realizó a los 14 días de edad, luego del registro de datos de peso corporal y consumo de alimento. Se indujo un estrés hídrico, donde las aves permanecieron 2 horas antes de la inoculación y 1 hora después sin acceso al agua de bebida. La inoculación fue directamente al buche mediante sonda rígida con $1 \mathrm{ml}$ de suspensión de ooquistes. Las aves sin desafiar fueron inoculadas con $1 \mathrm{ml}$ de agua destilada.

\section{Parámetros de Evaluación}

Se registraron los parámetros productivos desde la primera hasta la sexta semana de crianza. Se evaluó el peso corporal, la ganancia de peso, el consumo de alimento, el índice de conversión alimenticia (ICA), el índice de eficiencia productivo europeo (IEPE) y la viabilidad.

\section{Análisis de Datos}

El efecto de los tratamientos sobre cada parámetro productivo fue analizado mediante regresión lineal utilizando modelos lineales generalizados (GLM) con familia gaussiana y función de enlace identidad. El modelo de regresión quedó expresado de la siguiente manera: $\mathrm{E}(\mathrm{Y} \mid \mathrm{x})=\mathrm{g}(\mathrm{x})=\mathrm{b} 0+\mathrm{b} 1 \mathrm{X}$, donde $\mathrm{Y}$, representa el valor promedio esperado para cada parámetro productivo, b0 el intercepto (valor esperado de cada uno de los parámetros productivos cuando el efecto de los tratamientos es cero) y b1 corresponde a la pendiente o coeficiente de regresión para la variable $\mathrm{X}$ tratamiento (la diferencia promedio esperada para cada uno de los parámetros productivos al comparar cada uno de los tratamientos con uno referencial). La variable tratamiento $(\mathrm{k}=3)$ fue analizada como variable «dummy» en el análisis de regresión para obtener comparaciones entre pares de tratamientos. Por otro lado, la comparación entre los porcentajes de viabilidad según tratamientos fue analizada mediante la prueba no paramétrica de Kruskal Wallis. Valores p por debajo de 0.05 fueron considerados estadísticamente significativos.

\section{Resultados}

\section{A los 14 Días}

El peso promedio $(\mathrm{g})$ y ganancia acumulada de peso (g) a los 14 días y previo a la inoculación de cepas de Eimeria spp fueron similares entre tratamientos, lo que demuestra que las aves tenían las mismas condiciones antes del desafío experimental (Cuadro 1). El consumo de alimento acumulado e índice de conversión mostraron diferencia significativa entre los grupos G1 y G2 ( $<<0.05)$, pero no entre estos y el grupo control no tratado. 
Cuadro 1. Parámetros productivos de pollos machos de la línea Cobb 500 a los 14 días de edad ( $n=50$ aves por grupo)

\begin{tabular}{lccc}
\hline \multirow{2}{*}{ Parámetros productivos } & G1 & G2 & G3 \\
\cline { 2 - 4 } & Media \pm D.E. & Media \pm D.E. & Media \pm D.E. \\
\hline Peso promedio $(\mathrm{g})$ & $473.9 \pm 10.9^{\mathrm{a}}$ & $476.7 \pm 8.2^{\mathrm{a}}$ & $485.5 \pm 12.0^{\mathrm{a}}$ \\
Ganancia de peso acumulada $(\mathrm{g})$ & $427.7 \pm 10.9^{\mathrm{a}}$ & $429.1 \pm 8.3^{\mathrm{a}}$ & $437.7 \pm 12.5^{\mathrm{a}}$ \\
Consumo acumulado/ave $(\mathrm{g})$ & $526.9 \pm 25.3^{\mathrm{a}}$ & $555.2 \pm 18.7^{\mathrm{b}}$ & $548.4 \pm 14.8^{\mathrm{ab}}$ \\
Índice de conversión alimenticia & $1.11 \pm 0.05^{\mathrm{a}}$ & $1.17 \pm 0.05^{\mathrm{b}}$ & $1.13 \pm 0.02^{\mathrm{ab}}$ \\
\hline a,b Letras diferentes dentro de filas indican diferencias significativas entre tratamientos $(\mathrm{p}<0.05)$ \\
G1: tratado (salinomicina/nicarbazina 40 ppm) no desafiado; G2: tratado (salinomicina/nicarbazina 40 \\
ppm) desafiado; G3: no tratado y desafiado
\end{tabular}

Cuadro 2. Parámetros productivos de pollos machos de la línea Cobb 500 a los 21 días de edad ( $\mathrm{n}=50$ aves por grupo)

\begin{tabular}{lccc}
\hline \multirow{2}{*}{ Parámetros productivos } & G1 & G2 & G3 \\
\cline { 2 - 4 } & Media \pm D.E. & Media \pm D.E. & Media \pm D.E. \\
\hline Peso promedio $(\mathrm{g})$ & $908.7 \pm 17.0^{\mathrm{a}}$ & $853.3 \pm 14.0^{\mathrm{b}}$ & $768.4 \pm 12.6^{\mathrm{c}}$ \\
Ganancia de peso acumulada $(\mathrm{g})$ & $862.4 \pm 17.2^{\mathrm{a}}$ & $805.7 \pm 14.0^{\mathrm{b}}$ & $720.6 \pm 13.5^{\mathrm{c}}$ \\
Consumo acumulado/ave $(\mathrm{g})$ & $1072.5 \pm 37.5^{\mathrm{a}}$ & $1089.4 \pm 25.6^{\mathrm{a}}$ & $1007.8 \pm 27.9^{\mathrm{b}}$ \\
Índice de conversión alimenticia & $1.18 \pm 0.03^{\mathrm{a}}$ & $1.28 \pm 0.04^{\mathrm{b}}$ & $1.31 \pm 0.02^{\mathrm{b}}$ \\
\hline
\end{tabular}

$a, b$ Letras diferentes dentro de filas indican diferencias significativas entre tratamientos $(p<0.05)$

G1: tratado (salinomicina/nicarbazina 40 ppm) no desafiado; G2: tratado (salinomicina/nicarbazina 40 ppm) desafiado; G3: no tratado y desafiado

Cuadro 3. Parámetros productivos de pollos machos de la línea Cobb 500 a los 42 días de edad ( $\mathrm{n}=50$ aves por grupo)

\begin{tabular}{lccc}
\hline \multirow{2}{*}{ Parámetros productivos } & G1 & G2 & G3 \\
\cline { 2 - 4 } & Media \pm D.E. & Media \pm D.E. & Media \pm D.E. \\
\hline Peso promedio $(\mathrm{g})$ & $2650.4 \pm 33.0^{\mathrm{a}}$ & $2431.6 \pm 171.6^{\mathrm{b}}$ & $2111.6 \pm 114.9^{\mathrm{c}}$ \\
Ganancia de peso acumulada $(\mathrm{g})$ & $2604.1 \pm 32.4^{\mathrm{a}}$ & $2384.0 \pm 171.4^{\mathrm{b}}$ & $2063.8 \pm 115.1^{\mathrm{c}}$ \\
Consumo acumulado/ave $(\mathrm{g})$ & $4066.7 \pm 134.3^{\mathrm{a}}$ & $3900.5 \pm 147.0^{\mathrm{a}}$ & $3493.2 \pm 213.0^{\mathrm{b}}$ \\
Índice de conversión alimenticia & $1.53 \pm 0.04^{\mathrm{a}}$ & $1.61 \pm 0.11^{\mathrm{ab}}$ & $1.65 \pm 0.04^{\mathrm{b}}$ \\
Índice de eficiencia europeo & $408.67 \pm 7.95^{\mathrm{a}}$ & $355.94 \pm 52.05^{\mathrm{b}}$ & $282.0 \pm 29.29^{\mathrm{c}}$ \\
\hline
\end{tabular}

a,b Letras diferentes dentro de filas indican diferencias significativas entre tratamientos $(p<0.05)$

G1: tratado (salinomicina/nicarbazina 40 ppm) no desafiado; G2: tratado (salinomicina/nicarbazina 40 ppm) desafiado; G3: no tratado y desafiado 


\section{A los 21 Días}

Los parámetros productivos evaluados 7 días pos-desafío se observan en el Cuadro 2. Los tres grupos presentaron diferencias significativas en peso y ganancia de peso, siendo menor en el grupo desafiado y no tratado (G3) $(\mathrm{p}<0.05)$. Asimismo, el menor consumo de alimento fue en G3 ( $<<0.05)$, mientras que la mejor conversión alimenticia se observó en el grupo tratado no desafiado $(\mathrm{p}<0.05)$.

\section{A los 42 Días}

Los parámetros productivos evaluados a los 42 días de edad se observan en el Cuadro 3. Los grupos tratados con la combinación anticoccidial salinomicina/nicarbazina, desafiado y no desafiado, obtuvieron un peso promedio y ganancia de peso superior al grupo desafiado y no tratado $(\mathrm{p}<0.05)$, siendo estos valores, además, superiores en G1 en comparación a G2 $(\mathrm{p}<0.05)$. El menor consumo de alimento fue en G3 ( $p<0.05)$. El grupo tratado y no desafiado obtuvo el mejor índice de conversión alimenticia con diferencia estadística frente al resto de grupos evalua$\operatorname{dos}(\mathrm{p}<0.05)$, mientras que no hubo diferencia significativa entre el grupo tratado desafiado (G2) y el no tratado desafiado (G3). Por otro lado, el índice de eficiencia productivo europeo fue superior para los grupos tratados con la combinación anticoccidial en comparación con el grupo desafiado y no tratado $(\mathrm{p}<0.05)$.

Los grupos tratados con la combinación anticoccidial presentaron $100 \%$ de viabilidad frente al grupo no tratado y desafiado que obtuvo un $94 \%$ de viabilidad. La causa de mortalidad, corroborada a través de la necropsia de la evaluación macroscópica del intestino y con el raspado de la mucosa intestinal fue de coccidiosis. Si embargo, esta diferencia no fue estadísticamente significativa ante la prueba de Kruskal Wallis.

\section{Discusión}

Los pesos promedios de las aves de los tres grupos fueron similares hasta antes del desafío experimental con cepas locales de Eimeria spp a los 14 días de edad. Posteriormente y como consecuencia de la inoculación, se fueron observando diferencias entre grupos, notándose desde los 21 días hasta el final de la crianza un menor peso promedio y menor ganancia de peso en las aves no tratadas y desafiadas (G3).

Estudios previos han demostrado la eficacia de los productos anticoccidiales. Morrison et al. (1961) reportaron que la nicarbazina fue eficaz sobre la supervivencia, peso corporal y score de lesiones por coccidias en pollos de engorde criados en baterías, que fueron desafiados con un inóculo mixto de Eimweria acervulina, E. maxima, E. necatrix, E. Brunetti y E. tenella. Estos resultados son similares a los obtenidos en el presente trabajo, donde el control de la coccidiosis se vio reflejada en mejores parámetros productivos.

La eficacia de salinomicina (60 ppm) fue igualmente demostrada en un estudio comparativo, siendo igual o más eficaz que 100 ppm de monensina y 75 ppm de lasalocida en grupos desafiados con combinaciones de $E$. acervulina, E. mivati, E. maxima, E. necatrix, E. Brunetti y E. tenella (Migaki, 1979). Por otro lado, cuando se utilizó en concentraciones más bajas (44, 55 y 66 ppm), similares a la utilizadas en el presente estudio (40 ppm), no se observaron efectos adversos (Harms y Buresh, 1987).

Si bien los anticoccidiales ionóforos controlan la coccidiosis, también se han mencionado algunos efectos adversos como, por ejemplo, la depresión del consumo de alimento (Bafundo, 1994); sin embargo, esta condición no fue observada en el presente estudio de- 
bido a que la dosis de salinomicina utilizada en la combinación anticoccidial es menor a la que se utiliza en caso fuese sola (40 ppm); además de aprovechar el efecto sinérgico de este ionóforo con la nicarbazina.

La mejora en el rendimiento productivo en los grupos tratados con la combinación anticoccidial salinomicina/nicarbazina en el control de la coccidiosis fue similar a la reportada por Paredes y Quintero (2010). No obstante, León (2010) obtuvo menor peso final y mayor conversión alimenticia con la combinación anticoccidial salinomicina $36 \mathrm{ppm}$ y nicarbazina 28.8 ppm en comparación a la utilización de las mismas drogas por separado. Las variaciones de estos resultados con los obtenidos en el presente trabajo podrían deberse a la baja dosis de anticoccidial utilizado por este autor, lo que posiblemente impidió un buen control de la coccidiosis.

La mortalidad observada en el grupo no tratado y desafiado, si bien sin diferencia significativa con los grupos tratados, podría deberse al menor consumo de alimento, producto de la depresión, y a las lesiones ocasionadas por la coccidiosis. Estos parásitos generan una pérdida de la integridad intestinal, aumentando los gastos de mantenimiento y afectando negativamente el suministro de los nutrientes a las aves (Ferket, 2007). De otro lado, en los grupos tratados, las lesiones intestinales observadas en aves sacrificadas posterior al desafío fueron de grado leve, tal y como lo indica Pérez (2015) quien utilizó parte de los datos evaluados en este estudio.

Es conocida la susceptibilidad al estrés por calor en las aves suplementadas con nicarbazina, debido a un aumento de la tasa metabólica y al rápido desarrollo de hipertermia (Sumano y Gutiérrez, 2010); sin embargo, ninguno de los grupos tratados con la combinación anticoccidial presentó mortalidad por estrés por calor, aún cuando la crianza se realizó en meses de verano (febrero y marzo), probablemente debido a la baja dosis de nicarbazina usada en la dieta (40 ppm). Estos resultados evidencian la seguridad de la combinación anticoccidial empleada.
Finalmente, en relación al índice de eficiencia productiva, parámetro que engloba los datos de peso vivo, índice de conversión alimenticia y viabilidad, se pudo apreciar que las aves de los grupos tratados obtuvieron un mejor rendimiento que las aves no tratadas y desafiadas por lo que se demuestra que existe un importante sinergismo entre ambas drogas en el control de la coccidiosis aviar, sin mostrar efectos adversos (Hernández y Petrone, 2005); debido a que las combinaciones anticoccidiales permiten actuar, tanto en fases luminales como intracelulares, asegurando un mejor control de la enfermedad y un mejor rendimiento productivo.

\section{Conclusiones}

- Se demuestra la eficiencia de la combinación anticoccidial en el control de la coccidiosis aviar en pollos de engorde desafiados experimentalmente.

- El efecto sobre el control de la coccidiosis se vio reflejado en un mayor peso corporal (2431.6, 2111.6 g), mejor índice de conversión $(1.61,1.65)$ y mejor índice de eficiencia productiva (355.94, 282.0) en el grupo tratado/desafiado en comparación al grupo no tratado/desafiado, respectivamente.

\section{Literatura Citada}

1. Bafundo K. 1994. Trends in coccidiosis control: present considerations and future conerns. In: Proc XXXIII West PDC. Sacramento, California, USA.

2. Conway D, Mckenzie M. 2007. Poultry coccidiosis: Diagnostic and testing procedures. $3^{\text {rd }}$ ed. Iowa, USA: Blackwell Publishing. 164 p.

3. Dalloul R, Lillehoj H. 2006. Poultry coccidiosis: recent advancements in control measures and vaccine development. Expert Rev Vaccines 5: 143-163. doi: 10.1586/14760584.5.1.143 
4. Del Cacho E, Bosch M. 2014. Coccidiosis: la enfermedad, consecuencias y tratamiento. Selecciones Avícolas 56(2): 13-17.

5. Ferket PR. 2007. Controlling gut health without the use of antibiotics. Department of Poultry Science, North Carolina State University. [Internet]. Available in: http://citeseerx.ist.psu.edu/ viewdoc/download?doi=10.1.1.318.$7689 \&$ rep $=$ rep $1 \&$ type $=$ pdf

6. Glazer E, Cullen W, Frame G, Goudie A, Koss D, Olso J, Ricketts A, Tynan E, Walshe N, Wernaun W, Schaf T. 1993. Semduramicin: design and preparation of a new anticoccidial ionophore by semisynthesis and mutasynthesis. Dev Ind Microbiol 32: 133-139.

7. Harms R, Buresh R. 1987. Influence of salinomycin on the performance of broiler chicks. Poult Sci 66: 51-54.

8. Hernández V, Petrone G 2005. Sistema de producción animal. I. Aves. Vol II. Cap VII. México: UNAM. p 139-166.

9. Keshavarz K, McDougald L. 1982. Anticoccidial drugs: growth and performance depressing effects in young chickens. Poult Sci 6: 699-705. doi: 10.3382/ps.0610699

10. León N. 2010. Diferentes programas de coccidiostatos en el alimento del pollo de engorde sobre los parámetros productivos y mortalidad. Tesis de Médico Veterinario. México: Universidad de Michoacana de San Nicolás de Hidalgo. 35 p.

11. Migaki T, Chappel L, Babcock W. 1979. Anticoccidial efficacy of a new- polyether antibiotic, salinomycin, in comparison to monensin and lasalocid in battery trials. Poult Sci 58: 1192-1196. doi: 10.3382/ps.0581192

12. Morrison W, Ferguson A, Connell M, McGregor J. 1961. The efficacy of certain coccidiostats against mixed avian coccidial infections. Avian Dis 5: 222228. doi: $10.2307 / 1587630$

13. Paredes B, Quintero P. 2010. Determinación de la prevalencia de coccidiosis en las granjas avícolas de la parroquia Imbaya del cantón Antonio Ante y estudio del desempeño de tres tipos de drogas anticoccidiales en el control de protozoarios del género Eimeria durante la producción de pollos parrilleros. Tesis de Ingeniero Agropecuario. Ibarra, Ecuador: Pontificia Univ. Católica del Ecuador. 109 p.

14. Pérez J. 2015. Escore de lesiones intestinales macroscópicas de coccidias en pollos de engorde desafiados con cepas locales de eimerias y suplementados con un programa anticoccidial (salinomicina / nicarbazina). Tesis de Médico Veterinario. Lima: Univ. Nacional Mayor de San Marcos. 61 p.

15. Sumano H, Gutiérrez L. 2010. Antiparasitarios. En: Farmacología clínica en aves. $3^{\mathrm{a}}$ ed. México: Ed Interamericana. p 365-454.

16. Williams R. 1999. A compartmentalized model for the estimation of the cost of coccidiosis to the world's chicken production industry. Int J Parasitol 29: 1209-1229.(Footnotes) 